\title{
Web Surveys In Business Research: Use, Design And Construction Issues
}

David Olsen (Email: dolsen@b202.usu.edu), Utah State University

Pamela Keltyka (Email: pkeltyk@uakron.edu), The University of Akron

Sharon Kimmell (Email: skimmell@uakron.edu), The University of Akron

\begin{abstract}
Online surveys have the potential to dramatically improve the distribution of surveys, reduce the cost of administering surveys and increase survey response rates. This can lead to an overall improvement in the quality of the research being conducted. We first discuss the advantages and potential problems associated with online surveys. We then discuss survey website design issues and provide guidance regarding the use of various features. We also discuss several website construction considerations. Finally, we report on several viable approaches for building an online survey system, which range from inexpensive and technically challenging to expensive and easy to use and build.
\end{abstract}

\subsection{Introduction}

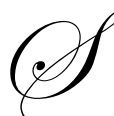

urvey research is sometimes the only way to investigate a particular issue, but data collection can be challenging. Mail surveys are not returned in a timely fashion and response rates are often low impacting the usability of some statistics. On-site or telephone data collection is difficult to schedule, costly, and often impractical for some broad-based types of research. In recent years, as a result of more widespread access to it by the general population, the Internet has provided researchers with another option in gathering survey data. In this paper, we discuss data collection using Internet technology.

The paper is divided into four main sections. The first section discusses the advantages of using the Internet for survey data collection while the second discusses the disadvantages and potential problems. The next section discusses survey and Website design issues, and the last discusses various construction versus purchase issues.

\subsection{Advantages in Using the Web for Survey Data Collection}

Cost Savings Survey data can be collected using the Internet at a much lower out-of-pocket cost per sample item than that of mail, in-person or telephone data collection. Compared to a mail survey, there are no printing or postage costs. Additionally, no one has to spend time stuffing envelopes or opening returned surveys. Compared to telephone or face-to-face interview data collection, the significant personnel costs, site costs and telephone charges are eliminated. Perhaps the most significant savings relates to data entry. If the Internet is used, no time has to be spent entering raw data into a database prior to analysis; this is certainly not the case with mail, telephone or face-to-face data collection. Geerts and Waddington (2002) reported on an Internet data collection tool that involved students studying database cardinalities. They found that the Internet is good tool for collecting data though issues such as a control over subjects can be a problem (pg. 14).

Ease of Administration Once the survey instrument is designed, the Website is constructed, and the target population has been identified, the administration of a Web survey is fairly simplistic. The individuals

$\overline{\text { Readers with comments }}$ or questions are encouraged to contact the authors via email. 
included in the sample can be invited via e-mail to participate in the survey. Follow-up requests can also be made easily through e-mail. The e-mail requesting completion of the survey should include a direct link to the survey instrument. This will eliminate the possibility of participants entering an incorrect Web address, being unable to access the survey, and perhaps quitting out of frustration.

Web surveys also allow for easier communication between the respondent and the researcher (Kiesler and Sproull, 1986). Participants are able to contact the researcher if they have problems accessing the survey or have questions or concerns about the instrument ((Bachmann, Elfrink, \& Vazzana, 1996; Mehta \& Sivadas, 1995). This can help to minimize measurement errors that result from a lack of understanding on the part of the respondent. This ability to get clarification about the test instrument or to voice concerns may also improve response rates.

Response Time Several studies have shown that data is returned significantly faster using a Web survey than a mail survey (Bachmann, Elfrink, \& Vazzana 1996; Sheehan \& McMillan, 1999; Sproull, 1986). Additionally, the researcher knows almost immediately which e-mail addresses are incorrect. This is certainly not the case with mail surveys. Having this information available early in the data collection process provides the opportunity to adjust the sample and thereby, enhance response rates (Weible \& Wallace, 1998).

Quality of Responses A survey instrument that includes open-ended questions may result in higher quality responses when administered via the Internet than by paper. Studies have found that Web survey participants give more candid and insightful responses and are more willing to respond to open ended questions than postal survey participants (Bachmann, Elfrink, \& Vazzana, 1996; Mehta \& Sivadas, 1995). Taylor (2000), states that he found replies to open-ended questions "richer, longer and more revealing" (p. 53). Kiesler and Sproull (1986) found that using an impersonal research setting, such as e-mail, made subjects more "unconcerned with social norms and with the impression they give others" and possibly willing to respond more honestly to the questions being asked than when surveyed using paper (p. 405).

Elimination of Data Input A significant advantage of electronic data collection is the elimination of the need to manually enter data from the returned surveys into a database or statistical package. The Website can be constructed so that responses are automatically entered into a database. Time must be invested prior to the data collection process in designing the database, but this time is well spent if it eliminates the manual entry process and the potential errors (data entry and inaccurate deciphering of handwriting) that accompany it.

\subsection{Potential Problems in Using the Web for Data Collection}

Reaching the Target Population A difficulty in administering a Web survey is reaching the target population. Although the number of people with access to the Internet has increased dramatically in recent years, a large percentage of the population still is not reachable if a Web survey is used. Schmidt (1997) encourages researchers to use Web surveys for populations with narrowly defined interests. For example, assume a researcher is interested in how one aspect of an audit is handled in a particular industry. If the population is comprised of auditors at the Big Four accounting firms, all of whom should have access to the Internet, a Web survey is feasible.

Respondent Confidentiality Issues When completing a postal survey, the respondent controls the confidentiality of the responses by making sure no identifying marks are left on the questionnaire. However, when submitting data through the Web, the respondent may feel that he/she no longer has the anonymity that exists with a mail survey. Therefore, in all correspondence, the researcher must reassure the participants that their anonymity will be maintained. Given that once participants submit the questionnaire they lose control, confidentiality may always remain an issue. Obviously, the more sensitive the data being gathered, the greater the impact this issue may have on response rates.

Legitimacy Issues Related to the issue of confidentiality is the issue of legitimacy of the researcher requesting participation in a survey. When a mail survey is used, the letter requesting participation is normally printed on letterhead of the researcher's sponsoring organization (university, corporation, etc.). Gathering data online and requesting participation via e-mail messages suppresses the cues used by participants to verify the 
legitimacy of the study (Sproull, 1986). To overcome a respondent's concerns, in the e-mail requesting participation, the researcher must include his or her complete identifying information including name, e-mail address, affiliate organization and address, and phone number. The e-mail message should encourage the participant to contact the researcher with any concerns or questions. If possible, providing the name of a contact within the researcher's organization who could verify the study and its purpose may also be helpful.

Response Rate Issues When collecting data using a survey, response rates are always a concern. Indeed, Odom et al. (1999) found that response rates were lower using email or World Wide Web surveys as compared to hard mail surveys. They experienced an $18.5 \%$ response rate with hard mail surveys as compared to $8 \%$ response rate on electronic surveys (pg. 31). Additionally, Hutchison et al. (2001) found that survey email response times are faster and that email is cheaper and more convenient than hard mail (pg.46). However, they also found that response rates were higher with hard mail surveys than email surveys.

One way to increase response rates when using a Web-based survey is to provide respondents with the option of completing a hard copy of the survey. This overcomes two potential problems. First, the respondent may be unable to complete the survey at the time the e-mail requesting participation is received and through oversight, may forget to access the survey at a later date. Second, some participants may not have time to complete the survey in a single sitting, may be interrupted during the process or may simply want to think about some of the questions prior to responding. Coming back to a paper survey at a later time is simple, but a Web survey doesn't easily provide this convenience. The difficulty in walking away from a partially completed Web survey and returning to complete it a later time may result in an incomplete survey or no survey being returned to the researcher. Allowing respondents to print and complete a hard copy of the survey may positively affect response rates. Just be sure to provide a fax number and address where a hard copy of the questionnaire can be returned.

Obviously, allowing participants to complete a hard copy of the survey negates the advantage of not having to input data. However, the increase in response rate that this option may create is probably worth the inconvenience of having to manually enter the responses from the hard copy to the survey instrument.

Control over Sample Another challenge in using a Web-based survey is ensuring that the respondents to the survey were actually included in the sample and that they only responded once. Several options exist to maintain control of the sample including passwords, e-mail addresses, qualifying questions and thank-you screens.

Requiring respondents to input a password in order to access the survey can eliminate multiple submissions (whether accidental or purposeful) from the same individual. Once the password has been used it will not allow access to the survey a second time (Kaye \& Johnson, 1999; Schmidt, 1997). Care must be taken in developing the password. Couper, Traugott, \& Lamias (2001) used alphanumeric passwords and found that passwords containing ambiguous characters, "the letters 1 [el] and o [oh] and the numbers 1 [one] and 0 [zero]" (p. 242) made participants significantly less likely to start the survey than those passwords that did not contain ambiguous characters.

Another way to eliminate multiple submissions is by having the respondent provide his/her e-mail address in response to the first question (Kaye \& Johnson, 1999). However, doing this could affect the respondent's need for anonymity and may result in the survey not being completed. Therefore, we advocate the use of a password as compared to an e-mail address.

To eliminate the problem of having the wrong person respond to the survey, a qualifier question can be used to screen participants (Schmidt, 1997). This device is especially useful when uncertainty exists about who actually received the e-mail requesting participation in the survey. For example, families may use one address for the entire family or friends may share an address; sharing makes it difficult to know who actually received the email and responded to the questionnaire. However, if a qualifier question is used, an individual incorrectly responding to the qualifier question can be deleted when reviewing the collected data.

Using a "thank you" screen can help eliminate accidental multiple submissions by a single individual who was uncertain as to whether the completed survey was successfully sent to the researcher. After the participant has 
clicked on the "return survey" or "submit survey" button, a "thank you" message verifying the successful submission of the survey should automatically appear (Kaye \& Johnson, 1999).

\subsection{Survey Design Issues}

First Page of the Questionnaire The first screen of the questionnaire may be the most important screen in the entire survey (Dillman, Tortora, \& Bowker, 1999). The main purpose of this screen is to inform respondents about how to access the contents of the questionnaire with as little effort as possible. It should not only verify that the respondent has reached the correct Website, but also motivate him or her to complete the survey.

Effective Instructions For a Web survey, the participant should have all of the pertinent instructions available on each screen. If the actions that are to be taken vary within the questionnaire, then instructions should be provided each time something different has to be done (Dillman, Tortora, \& Bowker, 1999). Once the instructions have appeared on one or two screens, they can probably be repeated in an abbreviated form or if they are fairly simple, even eliminated. Do not provide all the instructions at the beginning of the questionnaire and expect the respondent to refer back to them.

Dillman, Tortora, and Bowker (1999) suggest that completing a Web survey requires an individual to think simultaneously about operating the computer and correctly responding to survey questions. Consequently, providing complete, detailed, and effective instructions to the participants is critical. They suggest that the survey should instruct participants as to "when to click and double click with the mouse, when to use the return key, when and how to use a scroll bar, and how to change the size of windows" (p. 6).

Use of Images, Graphics and Color It is tempting to "dress up" a questionnaire when gathering data using a Web-based survey. Incorporating different font sizes, print styles, colors, graphics and images into the survey may be appealing, but before doing so, consider some of the problems this may cause your participants.

Related to the use of color, Dillman, Tortora, Conradt \& Bowker, (1998) tested the impact of two different types of surveys, which they labeled plain and fancy, on response rate. Their plain survey consisted of a white background with black printing while the fancy survey used alternating bands of bright pink and purple to highlight the questions. They report that the fancy survey did not result in a higher response rate when compared to the plain survey. They also found that the plain questionnaire took less transmission time and resulted in more of the questionnaire being completed than the fancy questionnaire.

Related to the use of images and graphics, Brennan, Rae, and Parackal (1999) found that depending upon their screen resolution, participants might have to use scroll bars to see the entire graph or picture which may alter the way they view the survey. Having to constantly scroll up or down and side-to-side may frustrate participates leading to incomplete surveys.

Another concern is the type of Internet connection the respondent is using (Solomon, 2001). Those individuals with slower Web access will require a longer time period to download large graphics. They may be unwilling to spend the time it takes to access the Website, and if they pay for their Internet access based upon connection time, the longer it takes to download the survey, the less willing they may be to participate (Kaye \& Johnson, 1999).

On a related note, in designing the Web questionnaire, consideration must be given to the variety of browsers and hardware available to survey participants. A line of text that fits comfortably on one browser might wrap into multiple lines on another, thus destroying the survey's formatting (Kaye \& Johnson, 1999). Another possibility is that a long line of text might not wrap properly forcing the participant to scroll out to the end of the line to see the entire question. The fancier the survey instrument, the more likely it is to cause problems for participants with older browsers. The browser is more likely to crash when downloading the survey and once the questionnaire is available it may have disabled response features (Dillman, Tortora, Conradt, \& Bowker, 1998). Obviously, if 
these problems are significant, some respondents may abandon the questionnaire perceiving it as more work than is necessary.

Remember, too, that not only will the extra "bells and whistles" make the survey instrument more difficult to use for some participants, but they also add to the cost of development of the survey (Dillman, Tortora, Conradt \& Bowker, 1998). It is important to examine whether the benefits of these extras justify the costs.

Use of Progress Indicators An individual receiving a mail survey needs only to skim through the survey to determine whether or not to participate. If an individual chooses to participate in the survey, he or she can, at any point in time, easily determine how much of the survey remains to be completed. Coupers, Tragott and Lamias (2001) argue that without progress indicator(s), especially in an interactive Web survey, participants don't know how much of the survey remains and may abandon the survey with only a small amount remaining. For a Web survey, several options exist to provide participants with information regarding their progress.

One option is to build into the design the ability for the individual to scroll through the entire survey at any point in time. However, this may not always be desirable or possible in the case of interactive surveys. Another is to place progress statements throughout the survey (i.e., you only have $25 \%$ of the survey left to complete or you only have ten questions remaining). Another possibility is to build into the survey a progress indicator.

Coupers, Tragott and Lamias (2001) tested the use of a progress indicator on the completion rates of a Web survey. They "implemented a graphic and text indicator in the upper right corner of every screen" (p. 3). Their results marginally supported the hypothesis that a higher percentage of those who had a progress indicator would complete the survey (p. 9). They also found that the surveys using the progress indicator took longer to complete. This may have been attributable to a lengthier download time or greater care taken by those completing the survey. The researchers were unsure of the explanation but speculated that a progress indicator would have a more significant effect in a study " where the progress indicator does not add to the download time (p. 9)."

Use of Radio Buttons, Response Boxes, Drop-down Box In designing the Web survey, a decision must be made as to how responses will be captured. Three options are available: radio buttons, drop-down boxes and response boxes. Radio buttons have one button for each response, and all of the buttons are simultaneously seen on the screen. A drop-down box requires the participant to click on the box to open and reveal all of the available responses. A response box is essentially a fill in the blank. The respondent composes the appropriate response in the designated area of the response box.

An advantage of radio buttons or drop-down boxes over response boxes is that they limit respondents' answers to only those that are valid (i.e., respondents can' t purposely or mistakenly input nonsense answers). Radio buttons and drop-down boxes do have a drawback if the range of answers is quite broad (the respondent's age, for example). In this situation, the researcher is forced to provide ranges for the respondent's answer (18 to 25,25 to 39 , etc.) since providing a radio button or single line in a drop-down box for every possible age (as many as 80 or more) would quickly make the survey unwieldy. Compared to radio buttons, drop-down boxes save space and clutter by concealing all of the available choices until the respondent clicks on the box (Kaye and Johnson, 1999).

Another drawback to using radio boxes or drop-down menus is simply the potential unfamiliarity of the respondents to such tools. Some survey participants may not know what has to be done to change an answer. Obviously, clear instructions would help to mitigate this problem.

Coupers, Tragott and Lamias (2001) tested the use of radio buttons versus response boxes. They hypothesized that data entry using radio buttons would take less time; however, their findings didn't support this hypothesis. They also found that more missing data resulted from the response box version of the questionnaire as compared to the radio button version. However, questions requiring the respondent to enter five numbers summing to ten were answered correctly more often by respondents assigned to the response box survey format than those using the radio button format. 
The choice of response format will depend to a large extent upon the needs of the researcher. However, as Coupers, Tragott and Lamias (2001) suggest, "relatively minor formatting changes could have an impact on the responses to a survey question (p. 10).”

Screen Composition Another issue that must be resolved in designing the Web survey is how much information to include on a single screen. Should a separate screen be devoted to each question or does it make more sense to put multiple questions on each screen? Coupers, Tragott and Lamias (2001, p. 7-8) speculated that grouping common items on a single screen would increase the correlation among the items. While the results of their study supported this notion, the differences were not statistically significant. They did find that questionnaires with multiple-question screens required significantly less time to complete than did those with single-question screens, and they resulted in fewer non-substantive responses.

\subsection{Website Construction Versus Acquisition Issues}

Website Construction Considerations If a survey Website is being constructed, then a number of items should be considered during the construction phase. First, as with any systems project, plenty of time should be allocated to build and test the site. The site should be tested on a variety of Web browsers and computer platforms. Special attention should be given to older Web browsers, older computers, and non-Windows computers like Macintosh's and UNIX-based computers. The system should be built using quality coding and extensive testing so that the system doesn't fail when respondents attempt to use it. "Quick and dirty Websites" can be built, but they fail more frequently. Obviously, this would affect response rates. Where possible, we advocate a minimalist programming approach so that speed is not compromised. As discussed in the design issues section, if survey respondents encounter a slow system, they will be less likely to complete the survey.

Second, great care should be given to ensuring good communication between technical and non-technical people on the project. The survey questions and answer types must be written in stone before the Website is even started since making changes to a Website after it is built and connected to a database can be very difficult and error prone. Also, determine what answers are expected (i.e. a 1,2,3 ... or $\mathrm{T}$ or $\mathrm{F}$ etc.), how the answers are to be collected (radio buttons, drop-down boxes or response boxes), and what the answers mean. Be sure to document all of the steps in the process because it is easy to forget the assumptions underlying the Website design and as a result, misinterpret an answer. For example, is a true answer represented internally to the computer as a 0 and a false as a 1? Understanding how an answer is coded is obviously crucial to reliable statistics. If strong communication is lacking, it is easy to make incorrect assumptions or to misinterpret the intent of the researcher.

Third, if a database is being constructed, it must be well designed to facilitate statistical analysis. A proper table structure, appropriate data types, and properly functioning keys (primary and foreign) are essential.

Finally, a robust Internet server or an excellent service provider must be used so that the system is always up. The response rate will be adversely affected if the server is down; especially during the first few days that the survey is announced since most attempts to respond will occur during this time period.

Survey Website Construction Like all information systems projects, the user interface design and the way the system works to facilitate the survey is crucial to a successful survey. It is axiomatic that some designs are more user-friendly than others, so a sound front-end design must be agreed upon early in the Website construction process. As discussed in the Website design section, survey participants are more likely to start and finish the survey if the system is elegant and user-friendly than if it is difficult to use.

Several tools and technologies that can be used to build an online survey Website are discussed below. They are compared in terms of the following factors (see Exhibit 1): 
1) Cost

2) Ease of Construction (degree of technical expertise required)

3) Flexibility (adaptability to a variety of circumstances)

4) Robustness (the ability to incorporate multiple features)

Cold Fusion is a proprietary Website building product. When combined with a good backend database, this product makes for an expensive, powerful, flexible, and relatively easy to build Website that facilitates data collection in the backend database.

A second common approach for Website construction involves Microsoft tools and technologies including active server pages (ASP), InterDev tools, or the new .NET approach. Each approach would be combined with a backend database such as Microsoft's SQL Server 2000. This approach is less expensive than the Cold Fusion approach and is relatively easy to use. However, it still requires extensive programming abilities so it is not as easy to use or as powerful as Cold Fusion.

A third approach includes open source tools that are freely available on the Internet. One example uses PHP (a recursive acronym standing for "PHP: Hypertext Preprocessor") combined with a backend database like MySQL or Postgres. The obvious advantage is that all of the software is free, but this is difficult to develop and harder to use. Additionally, a database like MySQL has few controls and security or management features. If a skilled open source developer is on the research team, this can be a cost effective approach.

Perhaps the most difficult and most powerful approach would include the open source tools Perl and Java performing Common Gateway Interface (CGI) tasks. These tools combined with a backend database such as MySQL or Postgres and a scripting language like Javascript are the most powerful in terms of allowing the survey designers the ability to custom program the survey so that nearly any functions can be implemented. These tools are free but provide the most difficult method of building a survey Website. Unless a member of the survey team has experience with these tools, we would advise against this approach.

Survey Website Acquisition Another approach to implementing the survey project requires little if any programming and simply involves researchers typing in questions and expected responses. This approach relies upon vendors that sell online survey tools as well as server space. When the survey is completed, the data can be collected and analyzed by the researchers. The following is a sample list of electronic survey vendor URL's:

http://www.ebreviate.com/

http://www.srbi.com/itools.htm

http://www.raosoft.com/products/tools/

This approach is the easiest but most expensive. It is also somewhat limited in the customized functions that can be implemented.

\subsection{Conclusion}

As a result of its ease of use and cost savings, the Internet's role in survey research has increased dramatically during the past decade. While this medium for delivery and completion of a survey is ripe with possibilities, researchers must be cognizant of the potential problems. Many of these problems can be avoided as long as significant care is exhibited during the Website design phase of the project. This paper discussed some of the relevant issues related to survey design and Website construction. Given the relative infancy of this form of survey research, numerous opportunities for additional research exist. While some studies have tested the effectiveness of various Website design features, in many cases, not enough research has been conducted in this area to be able to unequivocally support one design feature over another. 


\begin{tabular}{|l|c|c|c|c|}
\hline \multicolumn{5}{|c|}{ Exhibit 1: Comparison of Construction Option Characteristics } \\
\hline & Cost & $\begin{array}{c}\text { Ease of } \\
\text { Construction }\end{array}$ & Flexibility & Robustness \\
\hline Cold Fusion and Backend Database & H & H & H & H \\
\hline $\begin{array}{l}\text { Microsoft Tools and Technologies and Backend } \\
\text { Database }\end{array}$ & M & M & H & M \\
\hline Free Internet Sources and Backend Database & L & L & M & M \\
\hline $\begin{array}{l}\text { Open source tools Perl and Java and backend } \\
\text { database }\end{array}$ & L & L & H & H \\
\hline $\begin{array}{l}\text { L = low } \\
\text { M = moderate } \\
\text { H = high }\end{array}$ & & & \\
\hline
\end{tabular}

\section{References}

1. Bachmann, D., Elfrink, J., \& Vazzana, G. 1996. “Tracking the Progress of E-Mail Vs. Snail-Mail.” Marketing Research, 8: 31-35.

2. Brennan, M, Rae, N., \& Parackal, M. 1999. "Survey-based Experimental Research Via the Web: Some Observations." Marketing Bulletin, 10, 83-93.

3. Couper, M.P., Traugott, M.W., \& Lamias, M.J. 2001. "Web Survey Design and Administration.” Public Opinion Quarterly, 65: 230-254.

4. $\quad$ Dillman, D.A., Tortora, R.D., \& Bowker, D. 1999. "Principles for Constructing Web Surveys." Working paper available from http://survey.sesrc.wsu.edu/dillman/papers.htm [accessed 7-02].

5. Dillman, D.A., Tortora, R.D., Contradt, J., \& Bowker, D. 1998. "Influence of Plain Vs. Fancy Design on Response Rates for Web Surveys." Paper presented at the Joint Statistical Meetings of the American Statistical Association, Dallas and available from http://survey.sesrc.wsu.edu/dillmanpapers.htm [accessed 7-02].

6. $\quad$ Geerts, G.L., Waddington, B.A., 2002. "Design and Evaluation of An Internet Study Tool as a Data Collection Device." The Review of Business Information Systems, Vol. 6, Number 1:7-16.

7. Hutchison, P.D., Fleischman, G.M., Johnson, D.L., 2001. "Email Surveys: Additional Research Insights." The Review of Business Information Systems, Vol. 5, Number 2:37-48.

8. Kaye, B.K., \& Johnson, T.J. 1999. "Research Methodology: Taming the Cyber Frontier." Social Science Computer Review, 17: 323-337.

9. Kiesler, S. \& Sproull L.S. 1986. "Response Effects in the Electronic Survey.” Public Opinion Quarterly, 50: 402413.

10. Mehta, R. \& Sivadas, E. 1995. "Comparing Response Rates and Response Content in mail Versus Electronic Mail Surveys.” Journal of Market Research Society, 37: 429-439.

11. Odom, M.D., Giullian, M., Totaro, M.,1999. "New Technology in Survey Research: Does it Improve Response Rates?" The Review of Accounting Information Systems, Vol. 3, Number 1:27-34.

12. Schmidt, W.C. 1997. "World-Wide Web Survey Research: Benefits, Potential Problems, and Solutions." Behavior Research Methods, Instruments \& Computers, 29: 274-279.

13. Sheehan, K.B. \& McMillan, S.J. 1999. "Response Variation in E-Mail Surveys: An Exploration." Journal of Advertising Research:45-54.

14. Solomon, D.J. 2001. “Conducting Web-Based Surveys.” Practical Assessment Research \& Evaluation, 7, 19. Available from http://ericae.net/pare/getvn.asp?v=7\&n=19 [accessed 1-02].

15. Sproull, L.S. 1986. "Using Electronic Mail for Data Collection in Organizational Research." Academy of Management Journal, 29: 159-169.

16. Taylor, H. 2000. "Does Internet Research Work?” International Journal of Market Research, 42: 51-63.

17. Weible,R., \& Wallace, J. 1998. "Cyber Research: The Impact of the Internet on Data Collection." Marketing Research: 19-31. 